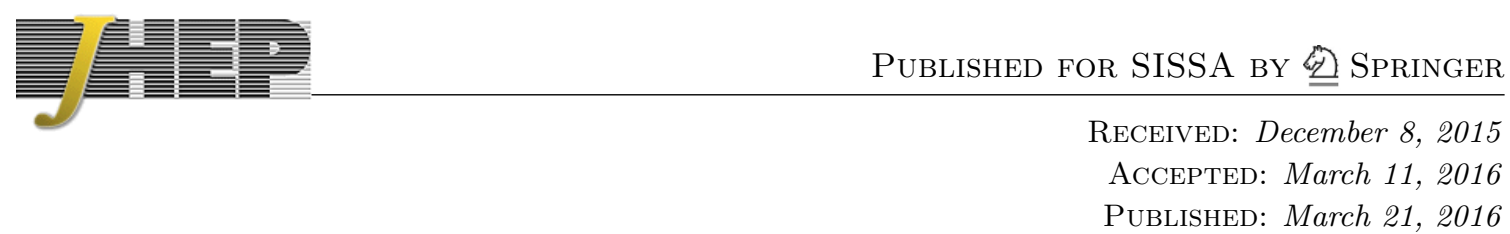

\title{
A nonperturbative proof of Dijkgraaf-Vafa conjecture
}

\section{Seiji Terashima}

Yukawa Institute for Theoretical Physics, Kyoto University, Kyoto 606-8502, Japan

E-mail: terasima@yukawa.kyoto-u.ac.jp

ABSTRACT: In this note we exactly compute the gaugino condensation of an arbitrary four dimensional $\mathcal{N}=1$ supersymmetric gauge theory in confining phase, using the localization technique. This result gives a nonperturbative proof of the Dijkgraaf-Vafa conjecture.

KEYWORDS: Supersymmetric gauge theory, Nonperturbative Effects, Solitons Monopoles and Instantons, Confinement

ARXIV EPRINT: 1509.02916 


\section{Contents}

1 Introduction and summary 1

2 Gaugino condensation in theory with a generic superpotential 2

$\begin{array}{lll}3 & \text { A proof of Dijkgraaf-Vafa conjecture } & 7\end{array}$

\section{Introduction and summary}

Analytic computations in quantum field theories are important, but very hard, in general. Important quantum field theories in which we can compute some quantities exactly are supersymmetric (SUSY) field theories. The localization technique for SUSY field theories, originated in $[1,2]$, is a general way to compute them exactly. Recently, this technique is applied to various kinds of SUSY field theories (for examples, [3]-[21]), after the important work by Pestun [22]. It should be stressed that using the localization technique we can compute non-topological quantities. In particular, using it, we can compute the gaugino condensation in four dimensional $\mathcal{N}=1$ SUSY Yang-Mills theories in confining phase [23]. ${ }^{1}$

In this paper, we will compute the gaugino condensation of four dimensional $\mathcal{N}=1$ SUSY gauge theories with general chiral multiplets and a superpotential in confining phase. In order to do this, we first integrate out the chiral multiplets, while keeping the vector multiplets. This is consistent because we can deform the theory without changing the gaugino condensation (i.e. using the localization technique) such that the theory is arbitrary weak coupling [23]. ${ }^{2}$ This integration of the chiral multiplets can be done perturbatively and we only need the effective superpotential. Thus, this can be done by the methods used in [31] and [32].

After the integration of the chiral multiplets, we have $\mathcal{N}=1$ SUSY gauge theories with only vector multiplets and a superpotential. As shown in [32], the superpotential is a function of the gaugino bi-linear $S$ only (and the coupling constants in the original superpotential). For this theory, we will compute the gaugino condensation. Therefore, we can compute the gaugino condensation in four dimensional $\mathcal{N}=1$ SUSY gauge theories with general chiral multiplets and a superpotential.

\footnotetext{
${ }^{1}$ The gaugino condensation were computed various ways, see [24]-[30].

${ }^{2}$ Here, we consider the theory on $\mathbf{R}^{3} \times S_{R}^{1}$ and then taking the $R \rightarrow \infty$ limit. (The gaugino condensation does not depend on $R$.) With the non-trivial v.e.v. of the Wilson line around $S_{R}^{1}$, the symmetry breaking will occur at the very high scale compared with the scale $\tilde{\Lambda}$ which is the effective dynamical scale determined by the deformed action. Thus, the computations reduced to $3 \mathrm{~d}$ Abelian theory in the low energy region much below the $1 / R$. Withtout this infra-red cut-off $R$, the deformed action will not be weak coupling because of the low energy modes below the $\tilde{\Lambda}$ which remain strong coupling.
} 
The Dijkgraaf-Vafa conjecture is that the glueball superpotential of the $\mathcal{N}=2$ SUSY $\mathrm{U}(N)$ gauge theory deformed by a superpotential is computed by a corresponding matrix model [33-35]. There are "proofs" of this conjecture, i.e. [31] and [32], however, both in [31] and [32] perturbative integrations of chiral multiplets were computed and the nonperturbative dynamics of the gauge fields were (implicitly) assumed to be just adding the Veneziano-Yankielowicz superpotential to the non-trivial superpotential which was obtained by the integration of the chiral multiplets. ${ }^{3}$ In this paper, we show that the perturbative superpotential with the Veneziano-Yankielowicz superpotential indeed gives the correct gaugino condensation. This can be regarded as a nonperturbative proof of the Dijkgraaf-Vafa conjecture. ${ }^{4}$

It should be noted that we can compute the gaugino condensation for any four dimensional $\mathcal{N}=1$ SUSY gauge theories (with a Lagrangian and in confining phase) according to the discussions in this paper. ${ }^{5}$ It would be interesting to study applications of our method. We hope to return this problems in future.

The organization of this paper is as follows: in section 2 we compute the gaugino condensation for four dimensional $\mathcal{N}=1$ SUSY gauge theory with only vector multiplets and a generic action. In section 3 we show that our results imply the nonperturbative proof of the Dijkgraaf-Vafa conjecture.

\section{Gaugino condensation in theory with a generic superpotential}

In this section, we will consider four dimensional $\mathcal{N}=1$ SUSY gauge theory with vector multiplets only (no chiral multiplets) on $\mathbf{R}^{3} \times S_{R}^{1}$ with a simple gauge group $G$ and the following superpotential:

$$
W_{V}\left(\tau_{0}, g_{i}\right)=2 \pi i \tau_{0} S+F\left(S, g_{i}\right),
$$

where $\tau_{0}$ and $g_{i}$ are complex constants, $S(y, \theta)=S_{0}(y)+\theta S_{1}(y)+\theta \theta S_{2}(y)$ is the glueball superfield whose lowest component is the gaugino bilinear $S_{0} \sim \operatorname{Tr}(\lambda \lambda)$ and $F\left(S, g_{i}\right)$ is a function of $S$ and coupling constants $g_{i}$. Here we do not assume the Kähler potential is canonical. Note that terms containing $\operatorname{Tr}\left((\lambda \lambda)^{n}\right)$ with $n>1$ and terms with derivatives are regarded as the Kähler potential [32]. Thus, this superpotential represents a general superpotential for a theory without chiral multiplets.

\footnotetext{
${ }^{3}$ More precisely, in [32] using the generalized Konishi anomaly equation to the 1PI effective action written by $S$ we can justify the addition of the Veneziano-Yankielowicz superpotential. However, as stressed in [32] this only works for the case without symmetry breaking because there are no coupling constants to $S_{i}$ where $S=\sum_{i} S_{i}$. We thank Y. Nakayama for the useful discussions on this point. In [32] it was also noted that the generalized Konishi anomaly would have the higher loop corrections.

${ }^{4}$ In [36], the gaugino condensation was computed in the way, which is different from ours and is related to the $\mathcal{N}=2$ Seiberg-Witten theory. The close connection of the gaugino condensation to Seiberg-Witten theory was also discussed in [37]. In [38], an off shell extension of the vacuum expectation value was used to compute the gaugino condensation and it was claimed to give a non-perturbative proof of the Dijkgraaf-Vafa conjecture.

${ }^{5}$ The perturbative superpotential should be computed using the results in [31] and [32] for general $\mathcal{N}=1$ gauge theories, for examples, [39, 40], however, it would be difficult to have the superpotential in an explicit closed form.
} 
Note that the polynomials of $S$ in the superpotential are composite operators and should be defined with a regularization, for example, a point splitting. Thus, the classical constraints are not imposed on these composites.

We will compute the effective superpotential for this theory and determine the vacua and then compute the gaugino condensation

$$
\bar{S} \equiv\langle S\rangle
$$

Using the result of [23], we can compute them in the weak Yang-Mills coupling constant by the localization technique. In a superspace, this is realized by adding

$$
t \int d^{4} x d^{2} \bar{\theta} \bar{W}_{\dot{\alpha}} \bar{W}^{\dot{\alpha}}
$$

to the anti-superpotential with $t \gg \infty$. The dynamical scale $\tilde{\Lambda}_{t}$ of the theory with the additional term (2.3) can be arbitrary low. ${ }^{6}$ With this deformed action, what we need to do is only semi-classical computations around the anti-self dual (ASD) connections. Note that the radius $R$ of $S_{R}^{1}$ plays as a infra-red regulator, which makes the deformed theory indeed weak coupling [23].

As in [23], in order to determine the vacua and evaluate the gaugino condensation, we would like to compute the expectation value of the gaugino bi-linear:

$$
\begin{aligned}
X \equiv\langle\lambda(x-b) \lambda(x)\rangle & =\left\langle\lambda(x-b) \lambda(x) e^{\int d^{4} y d^{2} \theta F\left(S(y, \theta), g_{i}\right)}\right\rangle_{0} \\
& =\left\langle\lambda(x-b) \lambda(x) e^{\int d^{4} y \delta_{1} \delta_{2} F\left(S_{0}(y), g_{i}\right)}\right\rangle_{0}
\end{aligned}
$$

where $\langle\cdots\rangle_{0}$ means the expectation value with the gauge coupling $\tau_{0}$ and a Kähler potential without the superpotential $F$. We also used $\delta_{1}, \delta_{2}$ which are SUSY transformations, corresponding to $\theta_{1}, \theta_{2}$. In order to evaluate this, it seems to have to consider ASD connections with many fermion zeromodes because the superpotential term $F$ includes the fermions. To do this explicitly is interesting, however, in this paper we will use a different method.

First, we introduce $\tilde{S}(x)$ as a constant shift $\bar{S}$ of $S(x)$ :

$$
\tilde{S}(x) \equiv S(x)-\bar{S},
$$

and define $G$ from $F$ by subtracting the zeroth and linear term in $\tilde{S}$ :

$$
G\left(\tilde{S}(x), g_{i}, \bar{S}\right) \equiv F\left(\bar{S}+\tilde{S}(x), g_{i}\right)-\left(\left.F(S)\right|_{S=\bar{S}}+\left.\frac{d F(S)}{d S}\right|_{S=\bar{S}} \tilde{S}(x)\right)
$$

Then, in terms of $\tilde{S}_{0}(x)$, we can express $X$ as

$$
X=\left\langle\lambda(x-b) \lambda(x) e^{\int d^{4} y \delta_{1} \delta_{2} G\left(\tilde{S}_{0}(y), g_{i}, \bar{S}_{0}\right)}\right\rangle_{\bar{S}}
$$

where

$$
\langle(\cdots)\rangle_{\bar{S}} \equiv\left\langle(\cdots) e^{\int d^{4} y \delta_{1} \delta_{2}\left(\left.F(S)\right|_{S=\bar{S}}+\left.\frac{d F(S)}{d S}\right|_{S=\bar{S}_{0}} \tilde{S}_{0}(y)\right)}\right\rangle_{0},
$$

\footnotetext{
${ }^{6}$ The dynamical scale can be arbitrary low by taking $t$ large enough depending on $\tau_{0}$ and $\langle S\rangle$.
} 
where we left $\left.F(S)\right|_{S=\bar{S}}$ term, which vanishes for constant $\bar{S}$, for later convenience. This term will be relevant if we regard $\bar{S}$ as a background constant chiral superfield. Note that the action of $\langle(\cdots)\rangle_{\bar{S}}$ contains only linear term in $\tilde{S}$.

Now we take the constant $\bar{S}$ to satisfy

$$
\bar{S}=\langle S(x)\rangle_{\bar{S}},
$$

which means $\langle\tilde{S}(x)\rangle_{\bar{S}}=0$. We will see later that this $\bar{S}$ indeed gives the gaugino condensation, i.e. $\bar{S}=\langle S(x)\rangle$. The condition (2.9) will be a self-consistent equation. ${ }^{7}$ Then we expand $e^{\int d^{4} y \delta_{1} \delta_{2} G\left(\tilde{S}_{0}(y), g_{i}, \bar{S}_{0}\right)}$ in $(2.7)$ in terms of $\tilde{S}_{0}$. It will be a linear combination of 1 and

$$
I_{n}=\int d^{4} x \delta_{1} \delta_{2}\left(C_{n}\left(S_{0}(x)\right)^{n}\right)
$$

where $C_{n}=C_{n}\left(\bar{S}_{0}, g_{i}\right)$ is determined by $G$. We can easliy see that $I_{n}$ satisfies $\bar{\delta}_{i} I_{n}=0$, because $\bar{\delta}_{i} S_{0}(x)=0$ and $\left[\bar{\delta}_{i}, \delta_{1} \delta_{2}\right]$ is a space derivative. Here $\bar{\delta}_{i}$ is the SUSY transformation corresponding to $\bar{\theta}_{i}$. Furthermore, we can show

$$
I_{n}=\int d^{4} x\left(\delta_{1} \delta_{2}\left(C_{n}\left(S_{0}(x)\right)^{n-1} e^{i a^{\mu} \partial_{\mu}} S_{0}(x)\right)+\bar{\delta}_{1} \delta_{1} \delta_{2}(\cdots)+\bar{\delta}_{2} \delta_{1} \delta_{2}(\cdots)\right) .
$$

This follows from $\frac{\partial}{\partial a_{\nu}} e^{i a^{\mu} \partial_{\mu}} S_{0}(x)=i\left(\bar{\delta} \sigma^{\mu} \delta\right) e^{i a^{\mu} \partial_{\mu}} S_{0}(x)$ which means $e^{i a^{\mu} \partial_{\mu}} S_{0}(x)=S_{0}(x)+$ $\left(\bar{\delta} \sigma^{\mu} \delta\right)(\cdots)$. Therefore, for the $\bar{\delta}_{i}$-closed correlators, which we are considering, we can replace

$$
I_{n} \rightarrow \int d^{4} x \delta_{1} \delta_{2}\left(C_{n} \prod_{j=1}^{n} S_{0}\left(x+a_{j}\right)\right)
$$

where $a_{j}$ is an arbitrary constant.

Note that we can do this replacement for each $I_{n}$ in a product of $I_{n} \mathrm{~s}$ in the expansion of the exponential with different $a_{j}$ for each $I_{n}$. Then, $X$ will be written by a linear combination of the following form:

$$
\left\langle\lambda(x-b) \lambda(x) \prod_{\alpha=1}^{M}\left(\int d^{4} x^{\alpha} \delta_{1} \delta_{2}\left(C_{n} \prod_{i=1}^{m_{\alpha}} \tilde{S}_{0}\left(x^{\alpha}+a_{i}^{\alpha}\right)\right)\right)\right\rangle_{\bar{S}}
$$

where $m_{\alpha} \geq 2$. Now we will take a following "large separations" limit: $\left|a_{j}^{\alpha}\right| \rightarrow \infty$ with $\left|a_{j}^{\alpha}-a_{k}^{\beta}\right| \rightarrow \infty((j, \alpha) \neq(k, \beta))$ and $\left|a_{j}^{\alpha}-b\right| \rightarrow \infty .^{8}$ Then, we will use the clustering properties to factorize the correlator for each $\left\langle\tilde{S}\left(x^{\alpha}+a_{i}^{\alpha}\right)\right\rangle$ if $\left|x^{\alpha}+a_{i}^{\alpha}\right|$ is not close to any other insertion points. Here we can see that $\left\langle\tilde{S}_{0}\left(x^{\alpha}+a_{i}^{\alpha}\right)\right\rangle_{\bar{S}}=0,\left\langle\delta_{i} \tilde{S}_{0}\left(x^{\alpha}+a_{i}^{\alpha}\right)\right\rangle_{\bar{S}}=0$ and $\left\langle\delta_{1} \delta_{2} \tilde{S}_{0}\left(x^{\alpha}+a_{i}^{\alpha}\right)\right\rangle_{\bar{S}}=0$ by the definition of $\tilde{S}$, i.e. $\langle\tilde{S}(x)\rangle_{\bar{S}}=0$. Furthermore, the number of the points of the integration is $M$, which is strictly smaller than the number of

\footnotetext{
${ }^{7}$ If we do not regard $\tau_{0}$ as a chiral superfield, we have $\langle S(x)\rangle=\left\langle S_{0}(x)\right\rangle$, i.e. $\left\langle S_{1}(x)\right\rangle=\left\langle S_{2}(x)\right\rangle=0$, because $S_{1}$ and $S_{2}$ are not Lorentz invariant.

${ }^{8}$ Here, we take the large $t$ limit (in (2.3)) such that $\left|a_{j}^{\alpha}\right| \tilde{\Lambda}_{t} \rightarrow 0$.
} 
$a_{i}^{\alpha}$ because $m_{\alpha} \geq 2$. Therefore, there is at least an isolated insertion of an operator which makes (2.13) vanishes and we find

$$
X=\left\langle\lambda(x-b) \lambda(x) e^{\int d^{4} y d \theta^{2} F\left(S(y, \theta), g_{i}\right)}\right\rangle_{0}=\langle\lambda(x-b) \lambda(x)\rangle_{\bar{S}}
$$

from which we can evaluate the superpotential, the vacua and the gaugino condensation as in $[23,41,42]$. Note that this also implies $\left\langle S_{0}(x)\right\rangle=\left\langle S_{0}(x)\right\rangle_{\bar{S}}$.

As we can see from $(2.8)$, the $\langle\cdots\rangle_{\bar{S}}$ is just replacing the coupling constant $\tau_{0}$ to

$$
\tilde{\tau}=\tau_{0}+\left.\frac{1}{2 \pi i} \frac{\partial F(S)}{\partial S}\right|_{S=\bar{S}} .
$$

Thus, the superpotential and the vacua ${ }^{9}$ are found as in [23, 41-43] by the semi-classical computations around the fundamental monopoles which have two fermion zeromodes. Note that the 1-loop factor in the localization technique only contributes to the Kähler potential $[44,45]$. More precisely, the definition of $\langle(\cdots)\rangle_{\bar{S}},(2.8)$, is the path-integral with the superpotential

$$
\tilde{W}=f(\bar{S})+2 \pi i \tilde{\tau} S
$$

where

$$
f(\bar{S})=\left.\left(F(S)-S \frac{\partial F(S)}{\partial S}\right)\right|_{S=\bar{S}}
$$

Thus, the effective superpotential is

$$
W_{\mathrm{eff}}=c_{2} \omega e(G) \tilde{\Lambda}^{3}+f(\bar{S})
$$

where $c_{2}$ is the dual coxeter number of $G$ and

$$
e(G)=\prod_{j=0}^{r}\left(k_{j}^{*} \alpha_{j}^{*} / 2\right)^{-k_{j}^{*} / 2}, \quad \omega^{c_{2}}=1,
$$

for example, $e\left(\operatorname{SU}\left(N_{c}\right)\right)=1$, and the $\tilde{\Lambda}$ is the dynamical scale in the 1-loop Pauli-Villars regularization which is defined by

$$
\tilde{\Lambda}^{3}=\mu^{3} \frac{1}{g^{2}(\mu)} \exp \frac{2 \pi \tilde{\tau}(\mu)}{c_{2}},
$$

where $g^{2}(\mu)$ comes from the path-integral measure which is defined with the coupling constant $\tau_{0}$.

Now we will consider the gaugino condensation $\langle S\rangle(=\bar{S})$. We have seen that the original superpotential $W_{V}$ can be written as

$$
W_{V}=f(\bar{S})+2 \pi i \tilde{\tau} S+G\left(\tilde{S}, g_{i}, \bar{S}\right),
$$

\footnotetext{
${ }^{9}$ In order to find the vacua, we need to evaluate $\partial W_{\text {eff }}\left(X, \tau_{0}\right) / \partial X=0$, where $X$ is the (would-be) moduli. With this and $\partial\left(W_{\text {eff }}\left(X, \tau_{0}\right)-f(\bar{S})\right) / \partial \tau_{0}=\bar{S}\left(X, \tau_{0}\right)$, we see that$$
\partial \bar{S}\left(X, \tau_{0}\right) / \partial X=0
$$

is a solution. Thus, we can think that $\bar{S}$ does not depend on $X$.
} 
then, the effective potential should give

$$
\frac{\partial}{\partial \tilde{\tau}} W_{\mathrm{eff}}(\tilde{\tau}, \bar{S}(\tilde{\tau}))=\left\langle 2 \pi i S+\frac{\partial}{\partial \tilde{\tau}} f(\bar{S})+\frac{\partial}{\partial \tilde{\tau}} G\right\rangle=\left\langle 2 \pi i S+\frac{\partial}{\partial \tilde{\tau}} f(\bar{S})\right\rangle,
$$

where we have used $\left\langle\frac{\partial}{\partial \tilde{\tau}} G\right\rangle=0$ which follows from $\langle($ polynomials of $\tilde{S})\rangle=0$. Thus, the gaugino condensation $\bar{S}$ can be computed using

$$
\frac{\partial}{\partial \tilde{\tau}}\left(W_{\mathrm{eff}}(\tilde{\tau}, \bar{S}(\tilde{\tau}))-f(\bar{S})\right)=\langle 2 \pi i S\rangle
$$

The result is

$$
\bar{S}=\langle S(x)\rangle=e(G) w \tilde{\Lambda}^{3} .
$$

The superpotential is evaluated to

$$
W_{\text {eff }}=c_{2} \bar{S}+f(\bar{S})
$$

where $\bar{S}$ is determined by (2.25). We will also define a dynamical scale $\Lambda$ in the 1-loop Pauli-Villars regularization of the coupling constant $\tau_{0}$,

$$
\Lambda^{3}=\mu^{3} \frac{1}{g^{2}(\mu)} \exp \frac{2 \pi \tau_{0}(\mu)}{c_{2}}
$$

The relation between $\Lambda$ and $\tilde{\Lambda}$ is given by

$$
\tilde{\Lambda}^{3}=\Lambda^{3} e^{\frac{1}{c_{2}} \frac{\partial F(\bar{S})}{\partial S}}
$$

where we have used $(2.15)$ and $\bar{S}=\bar{S}(\tilde{\Lambda})$ was given by $(2.25)$.

Now we see that the following glueball superpotential reproduces the gaugino condensation and the effective superpotential:

$$
W_{S}(S, \Lambda)=-c_{2} S\left(\ln \frac{S}{e(G) \Lambda^{3}}-1\right)+F(S)
$$

where we can think $W_{S}(S, \Lambda)$ as a function of $S$ and $\tilde{\Lambda}$ by using the relation $\tilde{\Lambda}=\tilde{\Lambda}(\Lambda)$. Indeed, we find

$$
\frac{\partial W_{S}}{\partial S}=-c_{2} \ln \frac{S}{e(G) \Lambda^{3}}+\frac{\partial F(S)}{\partial S}=0
$$

which is equivalent to $S=e(G) w \Lambda^{3} e^{\frac{1}{c_{2}} \frac{\partial F(S)}{\partial S}}$. The superpotential $W_{S}$ is evaluated with (2.30) to $W_{S} \rightarrow c_{2} S+F(S)-S \frac{\partial F(S)}{\partial S}$, which is the correct one. Therefore, the glueball superpotential is the (2.29) which is just a sum of the Veneziano-Yankielowicz superpotential and the $F(S)$.

We can easily generalize the results to the theory with a semi-simple gauge group. Let us consider a $4 \mathrm{~d} \mathcal{N}=1$ SUSY gauge theory of only vector multiplets with a gauge group $G=\otimes_{a} G_{a}$ is semi-simple and a superpotential

$$
W_{V}\left(\tau_{0}^{a}, g_{i}\right)=\sum_{a=1}\left(2 \pi i \tau_{0}^{a} S_{a}+F\left(S_{a}, g_{i}\right)\right) .
$$


Following the previous discussions, we can easily see that

$$
W_{S}\left(S_{a}, \Lambda\right)=-c_{2}^{a} \sum_{a} S_{a}\left(\ln \frac{S_{a}}{e\left(G_{a}\right) \Lambda_{a}^{3}}-1\right)+F\left(S_{a}\right)
$$

which is evaluated to $W_{S} \rightarrow c_{2}^{a} S_{a}+F\left(S_{a}\right)-\sum_{a} S_{a} \frac{\partial F\left(S_{a}\right)}{\partial S_{a}}$ with $S_{a}=e\left(G_{a}\right) w \Lambda_{a}^{3} e^{\frac{1}{c_{2}^{a}} \frac{\partial F\left(S_{a}\right)}{\partial S_{a}}}$. Here, for U(1) gauge group, there is no dynamically generated superpotential and $S_{a}=0$.

\section{A proof of Dijkgraaf-Vafa conjecture}

Let us consider $4 \mathrm{~d} \mathcal{N}=1$ gauge theory with gauge group $G$ and chiral multiplets couple to $G$. With a generic tree level superpotential

$$
W_{\text {tree }}=W_{\text {tree }}\left(g_{i}, \Phi_{a}\right),
$$

where $g_{i}$ is the coupling constants ${ }^{10}$ and $\Phi_{a}$ is the chiral superfields, the theory is expected to be in a confining phase ${ }^{11}$ and gaugino condensation is non-trivial, which we will compute.

We will compute the correlation functions of the operators insertions which satisfy $\bar{\delta}_{i}(\mathcal{O})=0$. Thus, we can add the regularization term of the localization for the vector multiplets (2.3) [23]. Then, the theory is effectively in weak coupling and the effective dynamical scale can be set to arbitrary low.

We can also add a large kinetic terms for the chiral multiplets. ${ }^{12}$ Then, we can integrate out the chiral multiplets perturbatively, where the vector multiplets are regarded as a background because the effective gauge coupling constant is very small by taking $t \rightarrow \infty$. Here we expand the bosonic fields in the chiral multiplets around the classical vacua. Note that the 1-loop computaiton is exact in the usual localization technique where we take the $t \rightarrow \infty$ limit with the regularization term $t \bar{\delta} V$ and rescaling of the fields. In our case, the kinetic terms of the chiral multiplets contain the vector multiplets which is regarded as background fields. Thus the saddle points of the large kinetic terms are nontrivial and integrations over the saddle points with the superpotential give a non-trivial effective superpotential. It will be interesting exactly follow this line and find the effective superpotential which should be a matrix model computation because the saddle points are essentially the zero modes of the chiral multiplets.

On the other hand, in [31] the perturbative computation of the chiral multiplets with the vector multiplet background was done by deforming the anti-superpotential appropriate way. Furthermore, in [32] it was shown that the effective superpotential obtained by integrating out the chiral superfields can be determined by the generalized Konishi anomaly.

\footnotetext{
${ }^{10}$ If the low energy theory is a non-trivial conformal fixed point, we will add an arbitrary small perturbation to the coupling constants or a small deformation of the vacuum we choose.

${ }^{11}$ With the chiral multiplets, the Wilson loop will not behave the are law. Thus, precisely speaking, the phase will not be a confining phase, but a phase with a mass gap with possible free U(1) factors. For simplicity, we will call it confining phase.

${ }^{12}$ The kinetic terms for the chiral superfields are written by the Kähler potential. The regularization term (2.3) for the vector multiplet is the anti-holomorphic superpotential. Both of them do not affect the effective superpotential and the correlation function of the operators in the chiral rings.
} 
Thus, in this paper, we assume that the integrating out the chiral multiplets is done by those methods.

Here, the chiral multiplets with classical superpotential can have a non-trivial moduli space of vacua. We have discrete set of vacua with a generic superpotential, although, the moduli space need not to be discrete. Here we assume the moduli space is discrete by giving a small deformation of superpotential, for example a mass term, if it is needed. Then, we redefine the chiral superfields as $\Phi_{a}^{\prime}=\Phi_{a}-\bar{\Phi}_{a}$ where $\bar{\Phi}_{a}$ is the value of $\Phi_{a}$ at the classical vacuum we have chosen. The perturbative calculation is done around this.

Depending on the choice of the classical vacuum, the original gauge group $G$ will be broken to a semi-simple gauge group with $\mathrm{U}(1)$ factors, which we will denote $G^{\prime}$. The glueball superfields $S_{a}$ are possible to be defined for this setting because the gauge symmetry is broken at very high energy scale compared to the effective dynamical scale of the gauge theory which is lowered by the regulator term. ${ }^{13}$ In terms of $S_{a}$, we have the effective superpotential $W_{V},(2.31)$, with $\tau_{0}^{a}=\tau_{0}$ for any $a$ after integration of the chiral superfields.

Then, applying the discussion in the previous section to the effective action (2.31), we conclude that the effective superpotential from which we can compute the gaugino condensation $\langle S\rangle=\sum_{a}\left\langle S_{a}\right\rangle$ is given by just adding the Veneziano-Yankielowicz superpotentials for all simple gauge groups in $G^{\prime}$ to the effective action (2.31).

In particular, if we consider a chiral multiplet of the adjoint representation of $G$, it was shown in [31] and [32] that the perturbative effective superpotential for the chiral multiplet is equivalent to the one of the matrix model of the Dijkgraaf and Vafa. Then, the pathintegral of the vector multiplets gives just the Veneziano-Yankielowicz terms according to the discussion in the previous section. The final effective action (2.31) is the one conjectured in [33-35] . Therefore, this can be regarded as a nonperturbative proof of the DijkgraafVafa conjecture. ${ }^{14}$

\section{Acknowledgments}

S.T. would like to thank Masato Taki for his collaboration at the early stage of this project and useful dicussions and Yu Nakayama for important comments and discussions. S.T. would like to thank K. Lee, K. Ohta, S. Rey, N. Sakai and P. Yi also for useful discussions.

Open Access. This article is distributed under the terms of the Creative Commons Attribution License (CC-BY 4.0), which permits any use, distribution and reproduction in any medium, provided the original author(s) and source are credited.

\footnotetext{
${ }^{13}$ The $S_{a}$ could be interpreted as a composite operator, like $\operatorname{Tr}\left(\Phi^{n} W_{\alpha} W^{\alpha}\right)$ where $\Phi$ is the chiral superfield, although we will not study this in this paper. Note that such relations are just for the expectation values. There is no effective superpotential fot $S_{a}$ for the original theory as stressed in [32] because corresponding coupling constants are absent.

${ }^{14}$ For the unbroken gauge group case, i.e. $G=G^{\prime}$, Dijkgraaf-Vafa conjecture was proved by using the generalized Konishi anomaly equation of the full effective action [32] and taking the decoupling limit to get the Veneziano-Yankielowicz terms.
} 


\section{References}

[1] E. Witten, Constraints on supersymmetry breaking, Nucl. Phys. B 202 (1982) 253 [INSPIRE].

[2] E. Witten, Topological quantum field theory, Commun. Math. Phys. 117 (1988) 353 [INSPIRE].

[3] A. Kapustin, B. Willett and I. Yaakov, Exact results for Wilson loops in superconformal Chern-Simons theories with matter, JHEP 03 (2010) 089 [arXiv:0909.4559] [INSPIRE].

[4] D.L. Jafferis, The exact superconformal R-symmetry extremizes Z, JHEP 05 (2012) 159 [arXiv:1012.3210] [INSPIRE].

[5] N. Hama, K. Hosomichi and S. Lee, Notes on SUSY gauge theories on three-sphere, JHEP 03 (2011) 127 [arXiv: 1012.3512] [INSPIRE].

[6] N. Hama, K. Hosomichi and S. Lee, SUSY gauge theories on squashed three-spheres, JHEP 05 (2011) 014 [arXiv: 1102.4716] [INSPIRE].

[7] F. Benini and S. Cremonesi, Partition functions of $\mathcal{N}=(2,2)$ gauge theories on $S^{2}$ and vortices, Commun. Math. Phys. 334 (2015) 1483 [arXiv:1206.2356] [INSPIRE].

[8] N. Doroud, J. Gomis, B. Le Floch and S. Lee, Exact results in D=2 supersymmetric gauge theories, JHEP 05 (2013) 093 [arXiv: 1206. 2606] [INSPIRE].

[9] K. Hosomichi, R.-K. Seong and S. Terashima, Supersymmetric gauge theories on the five-sphere, Nucl. Phys. B 865 (2012) 376 [arXiv:1203.0371] [INSPIRE].

[10] J. Källén and M. Zabzine, Twisted supersymmetric 5D Yang-Mills theory and contact geometry, JHEP 05 (2012) 125 [arXiv: 1202.1956] [INSPIRE].

[11] H.-C. Kim and S. Kim, M5-branes from gauge theories on the 5-sphere, JHEP 05 (2013) 144 [arXiv: 1206.6339] [INSPIRE].

[12] T. Kawano and N. Matsumiya, 5D SYM on 3D sphere and 2D YM, Phys. Lett. B 716 (2012) 450 [arXiv:1206.5966] [INSPIRE].

[13] J. Källén, J. Qiu and M. Zabzine, The perturbative partition function of supersymmetric 5D Yang-Mills theory with matter on the five-sphere, JHEP 08 (2012) 157 [arXiv:1206.6008] [INSPIRE].

[14] G. Festuccia and N. Seiberg, Rigid supersymmetric theories in curved superspace, JHEP 06 (2011) 114 [arXiv:1105.0689] [INSPIRE].

[15] S. Terashima, Supersymmetric gauge theories on $S^{4} \times S^{1}$, Phys. Rev. D 89 (2014) 125001 [arXiv:1207.2163] [INSPIRE].

[16] N. Hama and K. Hosomichi, Seiberg-Witten theories on ellipsoids, JHEP 09 (2012) 033 [arXiv:1206.6359] [INSPIRE].

[17] T. Nosaka and S. Terashima, Supersymmetric gauge theories on a squashed four-sphere, JHEP 12 (2013) 001 [arXiv:1310.5939] [INSPIRE].

[18] Y. Imamura, Relation between the $4 D$ superconformal index and the $S^{3}$ partition function, JHEP 09 (2011) 133 [arXiv: 1104.4482] [INSPIRE].

[19] Y. Asano, G. Ishiki, T. Okada and S. Shimasaki, Exact results for perturbative partition functions of theories with $\mathrm{SU}(2 \mid 4)$ symmetry, JHEP 02 (2013) 148 [arXiv:1211.0364] [INSPIRE]. 
[20] S. Sugishita and S. Terashima, Exact results in supersymmetric field theories on manifolds with boundaries, JHEP 11 (2013) 021 [arXiv:1308.1973] [INSPIRE].

[21] K. Hashimoto, S. Sugishita and S. Terashima, Ramond-Ramond couplings of D-branes, JHEP 03 (2015) 077 [arXiv: 1501.00995] [INSPIRE].

[22] V. Pestun, Localization of gauge theory on a four-sphere and supersymmetric Wilson loops, Commun. Math. Phys. 313 (2012) 71 [arXiv:0712.2824] [INSPIRE].

[23] S. Terashima, A localization computation in confining phase, JHEP 03 (2015) 097 [arXiv:1410.3630] [INSPIRE].

[24] M.A. Shifman and A.I. Vainshtein, On gluino condensation in supersymmetric gauge theories. $\mathrm{SU}(N)$ and $\mathrm{O}(N)$ groups, Nucl. Phys. B 296 (1988) 445 [InSPIRE].

[25] A. Yu. Morozov, M.A. Olshanetsky and M.A. Shifman, Gluino condensate in supersymmetric gluodynamics, Sov. Phys. JETP 67 (1988) 222 [Nucl. Phys. B 304 (1988) 291] [Zh. Eksp. Teor. Fiz. 94 (1988) 18] [inSPIRE].

[26] D. Finnell and P. Pouliot, Instanton calculations versus exact results in four-dimensional SUSY gauge theories, Nucl. Phys. B 453 (1995) 225 [hep-th/9503115] [INSPIRE].

[27] I. Affleck, M. Dine and N. Seiberg, Dynamical supersymmetry breaking in four-dimensions and its phenomenological implications, Nucl. Phys. B 256 (1985) 557 [inSPIRE].

[28] V.A. Novikov, M.A. Shifman, A.I. Vainshtein and V.I. Zakharov, Supersymmetric instanton calculus (gauge theories with matter), Nucl. Phys. B 260 (1985) 157 [InSPIRE].

[29] K.A. Intriligator and N. Seiberg, Lectures on supersymmetric gauge theories and electric-magnetic duality, Nucl. Phys. Proc. Suppl. 45BC (1996) 1 [hep-th/9509066] [INSPIRE].

[30] N. Seiberg, Exact results on the space of vacua of four-dimensional SUSY gauge theories, Phys. Rev. D 49 (1994) 6857 [hep-th/9402044] [INSPIRE].

[31] R. Dijkgraaf, M.T. Grisaru, C.S. Lam, C. Vafa and D. Zanon, Perturbative computation of glueball superpotentials, Phys. Lett. B 573 (2003) 138 [hep-th/0211017] [InSPIRE].

[32] F. Cachazo, M.R. Douglas, N. Seiberg and E. Witten, Chiral rings and anomalies in supersymmetric gauge theory, JHEP 12 (2002) 071 [hep-th/0211170] [INSPIRE].

[33] R. Dijkgraaf and C. Vafa, Matrix models, topological strings and supersymmetric gauge theories, Nucl. Phys. B 644 (2002) 3 [hep-th/0206255] [INSPIRE].

[34] R. Dijkgraaf and C. Vafa, On geometry and matrix models, Nucl. Phys. B 644 (2002) 21 [hep-th/0207106] [INSPIRE].

[35] R. Dijkgraaf and C. Vafa, A perturbative window into nonperturbative physics, hep-th/0208048 [INSPIRE].

[36] F. Fucito, J.F. Morales, R. Poghossian and A. Tanzini, $N=1$ superpotentials from multi-instanton calculus, JHEP 01 (2006) 031 [hep-th/0510173] [INSPIRE].

[37] M. Matone, Seiberg-Witten duality in Dijkgraaf-Vafa theory, Nucl. Phys. B 656 (2003) 78 [hep-th/0212253] [INSPIRE].

[38] F. Ferrari, Extended $N=1$ super Yang-Mills theory, JHEP 11 (2007) 001 [arXiv:0709.0472] [INSPIRE]. 
[39] A. Brandhuber, H. Ita, H. Nieder, Y. Oz and C. Romelsberger, Chiral rings, superpotentials and the vacuum structure of $N=1$ supersymmetric gauge theories, Adv. Theor. Math. Phys. 7 (2003) 269 [hep-th/0303001] [INSPIRE].

[40] Y. Nakayama, Effective gauge degrees of freedom and the (non)existence of the glueball superpotential, JHEP 08 (2003) 049 [hep-th/0306007] [INSPIRE].

[41] N.M. Davies, T.J. Hollowood, V.V. Khoze and M.P. Mattis, Gluino condensate and magnetic monopoles in supersymmetric gluodynamics, Nucl. Phys. B 559 (1999) 123 [hep-th/9905015] [INSPIRE].

[42] N.M. Davies and V.V. Khoze, On Affleck-Dine-Seiberg superpotential and magnetic monopoles in supersymmetric QCD, JHEP 01 (2000) 015 [hep-th/9911112] [INSPIRE].

[43] N.M. Davies, T.J. Hollowood and V.V. Khoze, Monopoles, affine algebras and the gluino condensate, J. Math. Phys. 44 (2003) 3640 [hep-th/0006011] [INSPIRE].

[44] E. Poppitz, T. Schäfer and M. Ünsal, Continuity, deconfinement and (super) Yang-Mills theory, JHEP 10 (2012) 115 [arXiv:1205.0290] [INSPIRE].

[45] M.M. Anber, E. Poppitz and B. Teeple, Deconfinement and continuity between thermal and (super) Yang-Mills theory for all gauge groups, JHEP 09 (2014) 040 [arXiv:1406.1199] [INSPIRE]. 